This item was submitted to Loughborough's Research Repository by the author.

Items in Figshare are protected by copyright, with all rights reserved, unless otherwise indicated.

\title{
Precise scenarios - a customer-friendly foundation for formal specifications
}

PLEASE CITE THE PUBLISHED VERSION

LICENCE

CC BY-NC-ND 4.0

\section{REPOSITORY RECORD}

Au, Oliver T.S., R.G. Stone, and John Cooke. 2019. "Precise Scenarios - a Customer-friendly Foundation for Formal Specifications". figshare. https://hdl.handle.net/2134/2647. 
This item was submitted to Loughborough's Institutional Repository by the author and is made available under the following Creative Commons Licence conditions.

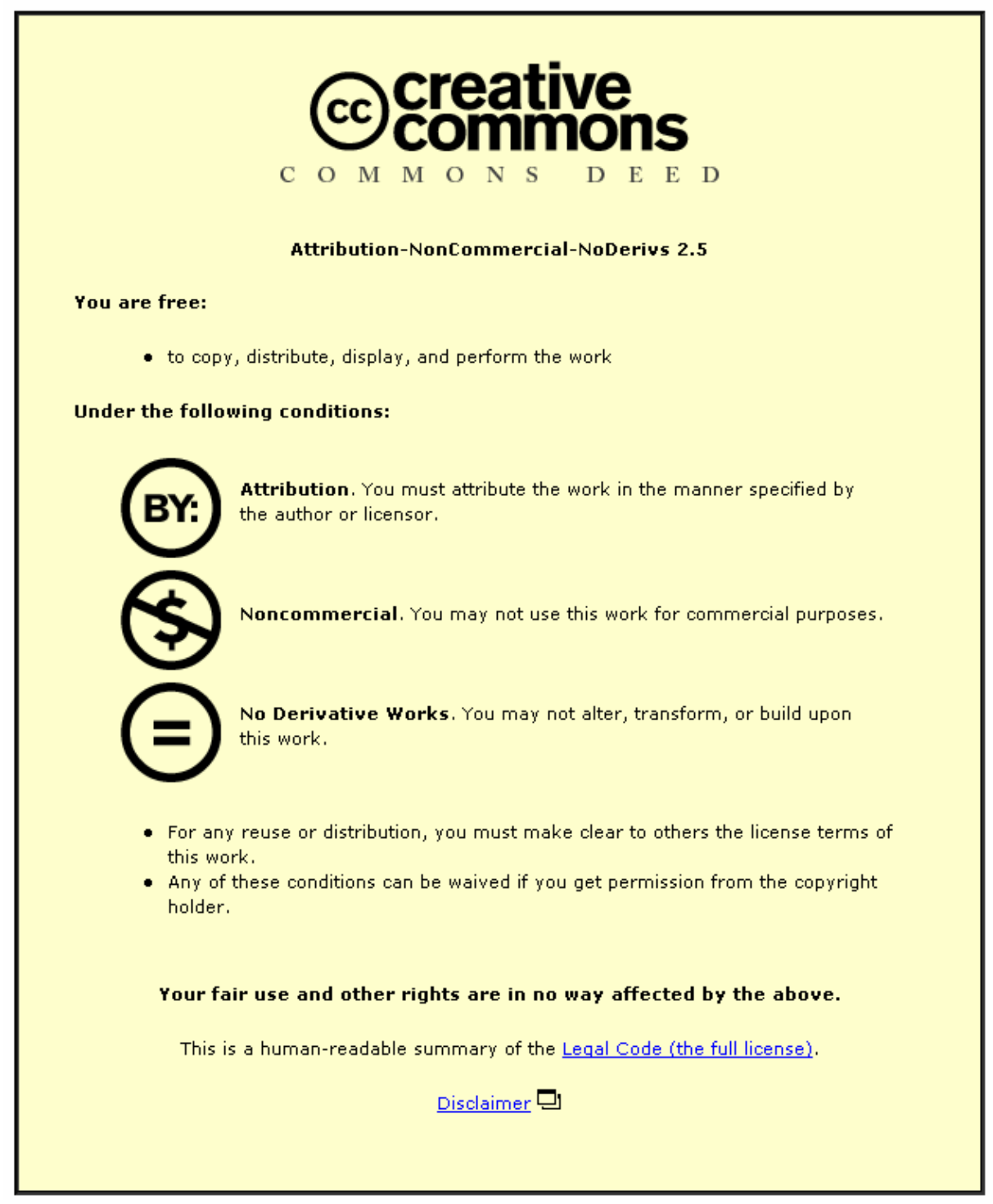

For the full text of this licence, please go to: http://creativecommons.org/licenses/by-nc-nd/2.5/ 


\title{
Precise Scenarios - A Customer-Friendly Foundation for Formal Specifications
}

\author{
Oliver Au, Roger Stone, and John Cooke \\ Loughborough University, England, \\ \{o.t.s.au, r.g.stone, d.j.cooke\}@lboro.ac.uk
}

\begin{abstract}
A formal specification is beyond the comprehension of the average software customer. As a result, the customer cannot provide useful feedback regarding its correctness and completeness. To address this problem, we suggest the formalism expert to work with the customer to create precise scenarios. A precise scenario describes an operation by its effects on the system state with only a few simple $\mathrm{Z}$ concepts. The customer would find a concrete precise scenario easier to understand than its corresponding abstract schema. The $\mathrm{Z}$ expert derives schemas based on the precise scenarios. Precise scenarios affords user involvement that improves the odds of a formal specification fully capturing the user requirements.
\end{abstract}

Key words: precise scenario, formal method, requirements specification, use case, $\mathrm{Z}$

\section{Introduction}

The most important software project success factor is user involvement [1]. Formal specification languages, being hard to read [2], discourage user involvement. The resulting formal specification may not truly reflect user's requirements. This could explain the limited adoption of formal methods [3]. We hope to broaden the appeal of formal specification by increasing user involvement with precise scenarios.

Use cases and scenarios may be used to involve customers in requirements elicitation. There should be a use case for each user task. A use case consists of several scenarios, one scenario for a particular situation. The most common way to describe the details of a scenario is by listing a sequence of steps. Each step describes an actor and its action in a natural language. Due to the ambiguous nature of natural languages, the formal specification cannot be verified against the scenario descriptions it was based on.

We propose to describe a scenario by its precise effects on a state. A state is represented by its actual data expressed in a small subset of the specification language $\mathrm{Z}$. For the layman, actual data are easier to understand than their abstract descriptions. The small number of $\mathrm{Z}$ symbols being used make our precise scenarios easier to understand than the corresponding schemas. The customers can participate in the creation of the precise scenarios. But scenarios only partially 
describe the infinite behaviour of a software application. A Z expert will need to generalise the scenarios into schemas that form a complete $\mathrm{Z}$ specification.

The idea of creating formal specifications from scenarios is not new. Amyot et al. expressed scenarios in Use Case Maps (UCM) to be translated into highlevel LOTOS specifications [4]. Whittle and Schumann started out with UML sequence diagrams and created statechart diagrams from them [5]. Uchitel et al. [6] and Damas et al. [7] used message sequence charts (MSC) to synthesize labeled transition system (LTS). Their work has been limited to system behaviour made up of sequences of parameterless events. Being based on $\mathrm{Z}$, we can extend sequences of simple events to general computation in the precise scenarios.

In another strand of research, Grieskamp and Lepper combined use cases with Z [8]. Use cases benefit from Z for its added precision while a Z specification benefits from use cases for relating its operations in actual usage. Test dialogues are built using nondeterministic choice, repetition and interruption. Executing a test dialogue in ZETA, the final state can be determined [9]. Their focus is on black-box test evaluation. The added capability has worsened the accessibility by software customers which contrasts with our research objective.

We use a simple ordering and invoicing problem to demonstrate our approach [10]. Section 2 describes the problem and a representation of the state space in Z. Each of sections 3 to 6 describes a user task, its precise scenarios and their use in the derivation of schemas. Section 7 briefly discusses validation, underspecification, overspecification, nondeterminism and tool support. Our conclusions are stated in section 8 .

\section{Ordering Problem and State Space}

There are four user tasks in our ordering problem: create a new order, invoice an order, cancel an order and refill the stock. The following statement introduces basic types OrderId and Product for the identification of individual orders and products. Once defined, we can use them in the specification without worrying about their implementation.

[OrderId, Product]

When an order is newly created, it will be in the state pending. After the order has left the warehouse, its state changes to invoiced. These are the only two order states that concern us regarding the scenarios to be discussed. The following definition could be modified by adding the new state paid to deal with payment scenarios in an iterative development process which is beyond the scope of this paper.

OrderState $::=$ pending $\mid$ invoiced

We declare the state space with schema OrderSystem which has four variables, and after a horizontal dividing line, two invariants. 


\begin{tabular}{l} 
OrderSystem \\
stock $:$ bag Product \\
orders $:$ OrderId $\rightarrow$ bag Product \\
orderStatus $:$ OrderId $\rightarrow$ OrderState \\
freeIds $: \mathbb{P}$ OrderId \\
\hline dom orders $=$ dom orderStatus \\
dom orders $\bigcap$ freeIds $=\varnothing$
\end{tabular}

A bag of Product is equivalent to a partial function from Product to the set of positive natural numbers $\mathbb{N}_{1}$. We use the function to keep track of a product's quantity in stock or in an order. The symbols $\rightarrow$ and $\mathbb{P}$ represent partial function and power set respectively.

In an arbitrary state, we have 5 nuts and 6 bolts in stock. Order 1 was placed for 2 nuts and 2 bolts. Order 2 was placed for 3 bolts. Order 1 has been invoiced and order 2 is still pending. Ids 3 and 4 are free for future use. The state could be expressed with the following values in the variables of schema OrderSystem.

$$
\begin{aligned}
& \text { stock }=\{\text { nut } \mapsto 5, \text { bolt } \mapsto 6\} \\
& \text { orders }=\{1 \mapsto\{\text { nut } \mapsto 2, \text { bolt } \mapsto 2\}, 2 \mapsto\{\text { bolt } \mapsto 3\}\} \\
& \text { orderStatus }=\{1 \mapsto \text { invoiced }, 2 \mapsto \text { pending }\} \\
& \text { freeIds }=\{3,4\}
\end{aligned}
$$

The keyword dom stands for domain. The first invariant ensures that an order id in use must appear in both orders and orderStatus for an order cannot exist without its status. The second invariant prevents an order id from being used and at the same time available for new orders. Often, we don't know all the constraints until we have explored the scenarios.

After an operation, we want to report whether it was successful. If not, what was the error?

$$
\begin{aligned}
\text { Report } & := \\
\mid & \text { OK } \mid \text { no_more_ids } \mid \text { order_not_found } \mid \text { not_enough_stock }
\end{aligned}
$$

\section{New Order}

The scenario NewOrder documents a successful order creation using four Z concepts. They are input with the symbol ?, output with!, maplet with $\mapsto$, and set with \{\} and commas. The input parameter order? places a new order for 4 nuts and 4 bolts. Below the table headings are the pre-state followed by the post-state. In the post-state, the 3-dot symbol ... is used to denote the unchanged function stock. Functions orders and orderStatus are extended by a map for OrderId 3. The element 3 is removed from the set freeIds. The output parameters $i d$ ! and report! return 3 and $O K$ respectively. 
scenario NewOrder

order $?=\left\{\right.$ nut $_{a} \mapsto 4_{b}$, bolt $\left._{c} \mapsto 4_{d}\right\}$

\begin{tabular}{|c|c|c|c|}
\hline stock & orders & orderStatus & freeIds \\
\hline $\begin{aligned}\{\text { nut } & \mapsto 5 \\
\text { bolt } & \mapsto 6\}\end{aligned}$ & $\begin{aligned}\{1 & \mapsto\{\text { nut } \mapsto 2, \text { bolt } \mapsto 2\}, \\
2 & \mapsto\{\text { bolt } \mapsto 3\}\}\end{aligned}$ & $\begin{aligned}\{1 & \mapsto \text { invoiced } \\
2 & \mapsto \text { invoiced }\}\end{aligned}$ & $\left\{3_{e}, 4\right\}$ \\
\hline$\cdots$ & $\begin{aligned}\{1 & \mapsto\{\text { nut } \mapsto 2, \text { bolt } \mapsto 2\} \\
2 & \mapsto\{\text { bolt } \mapsto 3\} \\
3_{e} & \left.\mapsto\left\{\text { nut }_{a} \mapsto 4_{b}, \text { bolt }_{c} \mapsto 4_{d}\right\}\right\}\end{aligned}$ & $\begin{array}{l}\{1 \mapsto \text { invoiced } \\
2 \mapsto \text { invoiced } \\
\left.3_{e} \mapsto \text { pending }\right\}\end{array}$ & $\{4\}$ \\
\hline
\end{tabular}

$i d !=3_{e}$, report $!=O K$

Values referred in input/output parameters are subscripted allowing us to relate them to the state. When two pieces of data have the same subscript, for example, $3_{e}$ in the post-state of orders and orderStatus, they must be identical. If two pieces of data have identical value but different subscripts, for example $4_{b}$ and $4_{d}$, their equality is merely a coincidence. Value $4_{d}$ could have been $5_{d}$ throughout the scenario. The values allowed in a scenario are confined by earlier definitions. For example, $4_{b}$ and $4_{d}$ must be taken from the set of positive natural numbers $\mathbb{N}_{1}$.

To generalise the above scenario to an operation schema, we need a type for the new order that maps Product to a positive integer.

$$
\text { Order }==\{\text { order }: \text { bag Product } \mid \text { order } \neq \varnothing\}
$$

The scenario NewOrder can be converted to the equivalent Z schema below. The declaration part declares the variables, input/output parameters and their types. The symbol $\Delta$ alerts us that the state of OrderSystem is changed by this operation. The predicate part lists the constraints on the variables and parameters. The trailing symbol ' is used to denote a value after the operation.

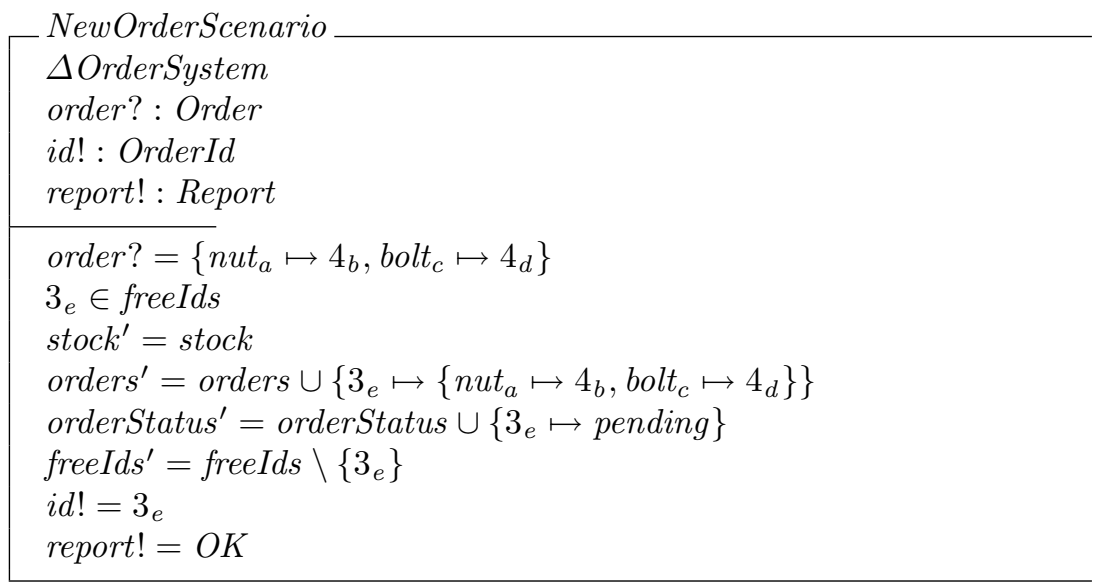

The first predicate specifies the value of the input parameter order?. The membership of $3_{e}$ in set freeIds gives rise to the second predicate. The third predicate states that stock is unchanged after the operation. The new maplets 
for $3_{e}$, adding to orders and orderStatus, are captured in the fourth and fifth predicates. The removal of $3_{e}$ from the set freeIds is expressed next. The values for the output parameters are specified by the last two predicates.

Subscripted values, used to represent input/output parameters, are not fixed. For example, instead of picking $3_{e}$ in the pre-state, we could have picked $4_{e}$. By replacing the subscripted values with the input/output variables they represent, for example, " $3_{e}$ " with " $i d !$ " and " $\left\{\right.$ nut $_{a} \mapsto 4_{b}$, bolt $\left._{c} \mapsto 4_{d}\right\}$ " with "order?", we have the generalised schema below.

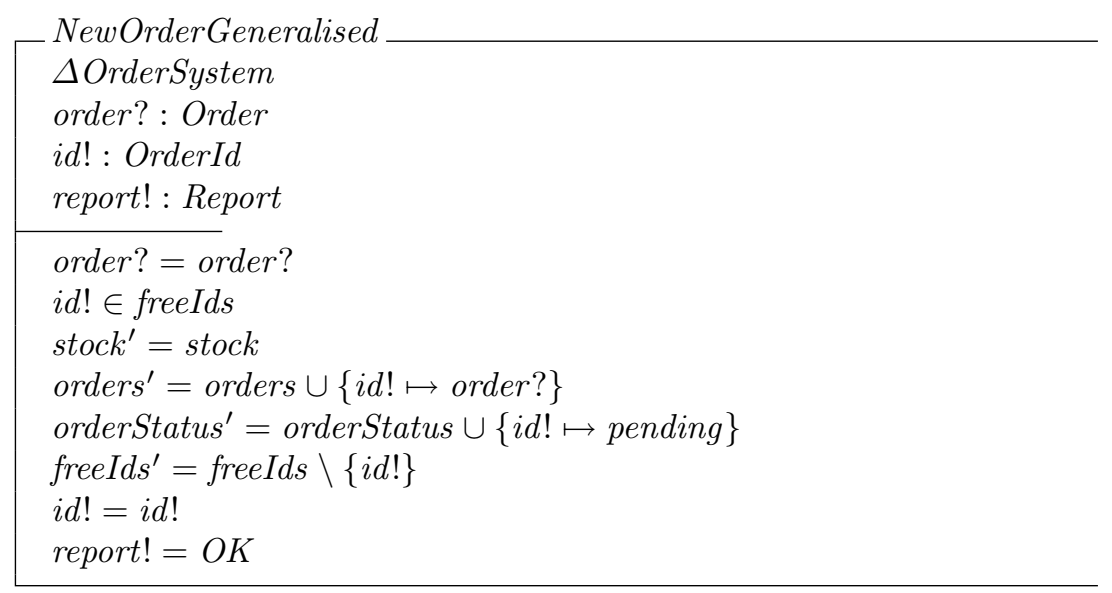

The generalised version of the $\mathrm{Z}$ schema can be simplified by removing the two identity predicates that always evaluate to true.

\begin{tabular}{|l} 
NewOrder \\
$\Delta$ OrderSystem \\
order? : Order \\
$i d !:$ OrderId \\
report $!:$ Report \\
$i d ! \in$ freeIds \\
stock ${ }^{\prime}=$ stock \\
orders $^{\prime}=$ orders $\cup\{$ id $! \mapsto$ order $\}$ \\
orderStatus ${ }^{\prime}=$ orderStatus $\cup\{$ id! $\mapsto$ pending $\}$ \\
freeIds $=$ freeIds $\backslash\{$ id $!\}$ \\
report $!=$ OK
\end{tabular}

We now turn our attention to an unsuccessful attempt to create a new order. A separate post-state is not shown in the scenario because the state is unchanged. No subscripts are used because the input/output parameters do not relate to any piece of data in the state. The pre-condition is the absence of any OrderIds in freeIds. 
scenario NoMoreIdsError

order $?=\{$ nut $\mapsto 7\}$

\begin{tabular}{|c|c|c|}
\hline \multicolumn{3}{|c|}{\begin{tabular}{l|l|l|l|} 
stock & orders & orderStatus & freeIds \\
$\{$ nut $\mapsto 5$, & $\{1 \mapsto\{$ nut $\mapsto 2$, bolt $\mapsto 2\}$, & $\{1 \mapsto$ invoiced,\{\}
\end{tabular}} \\
\hline $\begin{aligned}\{\text { nut } & \mapsto 5 \\
\text { bolt } & \mapsto 6\}\end{aligned}$ & $\begin{aligned}\{1 & \mapsto\{\text { nut } \mapsto 2, \text { bolt } \mapsto 2\}, \\
2 & \mapsto\{\text { bolt } \mapsto 3\}, \\
3 & \mapsto\{\text { nut } \mapsto 4, \text { bolt } \mapsto 4\}, \\
4 & \mapsto\{\text { bolt } \mapsto 8\}\}\end{aligned}$ & $\begin{aligned}\{1 & \mapsto \text { invoiced, } \\
2 & \mapsto \text { invoiced }, \\
3 & \mapsto \text { pending, } \\
4 & \mapsto \text { pending }\}\end{aligned}$ \\
\hline
\end{tabular}

report! =no_more_ids

The symbol $\Xi$ indicates that the state OrderSystem is unchanged by the schema.

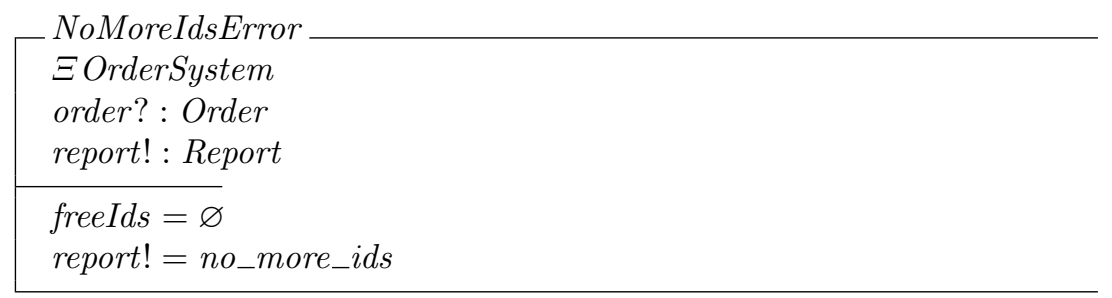

The pre-condition of NewOrder is that there is some element in freeIds. Conversely, the pre-condition of NoMoreIdsError is that the set freeIds is empty. The disjunction of the two pre-conditions is true. Therefore NewOrderOp can handle all situations.

$$
\text { NewOrderOp }==\text { NewOrder } \vee \text { NoMoreIdsError }
$$

\section{Invoice Order}

The invoicing operation updates the order status from pending to invoiced and reduces the stock accordingly. In the next scenario, we use subscripts to help us express constraints on data values in the state. The two pre-conditions, shown before the table, require the state to have sufficient stock to fill the order. The two post-conditions, shown after the table, determine the updated stock quantities.

scenario InvoiceOrder

$i d ?=2_{a}, 4_{f} \leq 5_{c}, 3_{h} \leq 9_{e}$

\begin{tabular}{|l|l|l|l|}
\hline stock & orders & orderStatus & freeIds \\
\hline nut $_{b} \mapsto 5_{c}$, & $\{1 \mapsto\{$ nut $\mapsto 2\}$, & $\{1 \mapsto$ invoiced, & $\{3,4\}$ \\
bolt $\left._{d} \mapsto 9_{e}\right\}$ & $2_{a} \mapsto\left\{\right.$ nut $_{b} \mapsto 4_{f}$, bolt $\left.\left._{d} \mapsto 3_{h}\right\}\right\}$ & $2_{a} \mapsto$ pending & \\
\hline$\left\{\right.$ nut $_{b} \mapsto 1_{i}$, & $\ldots$ & $\{1 \mapsto$ invoiced, & $\ldots$ \\
bolt $\left._{d} \mapsto 6_{j}\right\}$ & & $2_{a} \mapsto$ invoiced $\}$ & \\
\hline
\end{tabular}

report $!=O K, 1_{i}=5_{c}-4_{f}, 6_{j}=9_{e}-3_{h}$

The $\mathrm{Z}$ mathematical toolkit provides the sub-bag symbol $\sqsubseteq$ that concisely expresses multiple $\leq$ relationships between corresponding quantities in two bags. 
Likewise, the bag difference symbol $\uplus$ expresses multiple pairwise subtractions. The updating of the order status to invoiced is expressed with the override symbol $\oplus$. Round brackets represent function application, for example, orders $\left(2_{a}\right)$ returns $\left\{\right.$ nut $_{b} \mapsto 4_{f}$, bolt $\left._{d} \mapsto 3_{h}\right\}$.

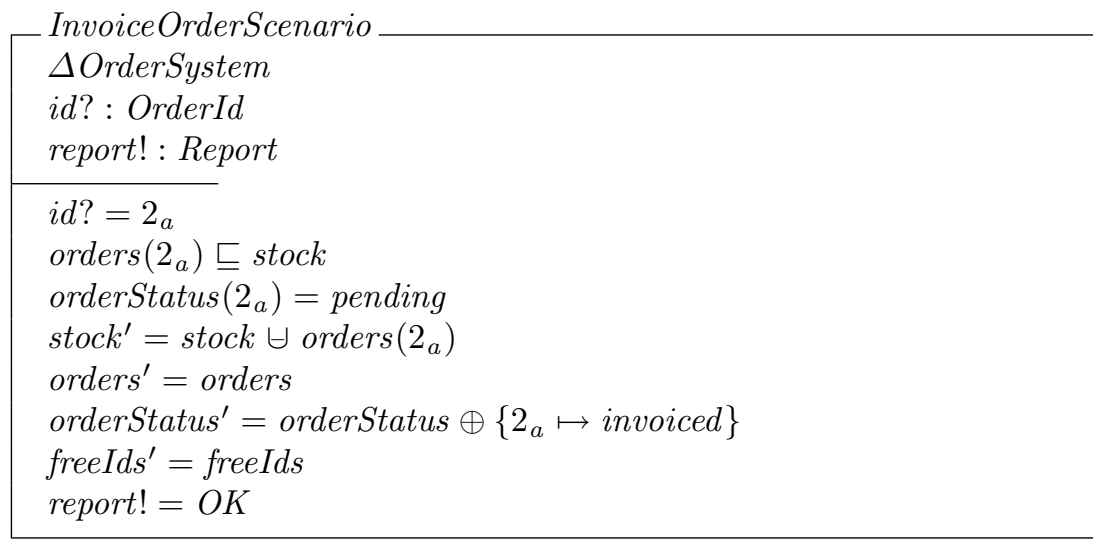

After substitution and simplification, we have the following $\mathrm{Z}$ schema.

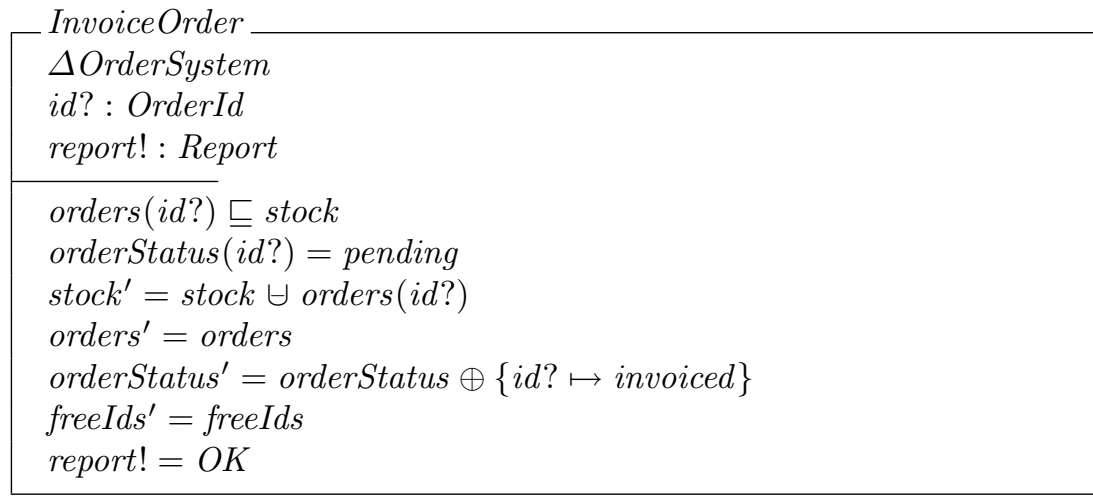

There are three unsuccessful scenarios for this operation. We will skim the intermediate steps and explanations of the remaining derivations due to their similarities to the previous ones.

scenario IdNotFoundError

$i d ?=3_{a}, 3_{a} \neq 1_{b}, 3_{a} \neq 2_{c}$

\begin{tabular}{|l|l|l|l|}
\hline stock & orders & orderStatus & freeIds \\
\hline nut $\mapsto 5$, & $\{1 \mapsto\{$ nut $\mapsto 2$, bolt $\mapsto 2\}$, & $\left\{1_{b} \mapsto\right.$ invoiced, $\{\{3,4\}$ \\
bolt $\mapsto 9\}$ & $2 \mapsto\{$ bolt $\mapsto 3\}\}$ & $2_{c} \mapsto$ pending $\}$ & \\
\hline
\end{tabular}


IdNotFoundErrorScenario

$\Xi$ State

id? : OrderId

report! : Report

$i d ?=3_{a}$

$3_{a} \notin\left\{1_{b}, 2_{c}\right\}$

report! = id_not_found

— IdNotFoundError

$\Xi$ State

$i d ?:$ OrderId

report! : Report

$i d ? \notin$ dom orderStatus

report $!=i d \_n o t \_f o u n d$

scenario OrderNotPendingError

$i d ?=1_{a}$, invoiced $_{b} \neq$ pending

\begin{tabular}{|l|l|l|l|}
\hline stock & orders & orderStatus & freeIds \\
\hline$\{$ nut $\mapsto 5$, & $\{1 \mapsto\{$ nut $\mapsto 2$, bolt $\mapsto 2\}$, & $\left\{1_{a} \mapsto\right.$ invoiced $_{b},\{3,4\}$ \\
bolt $\mapsto 9\}$ & $2 \mapsto\{$ bolt $\mapsto 3\}\}$ & $2 \mapsto$ pending $\}$ & \\
\hline
\end{tabular}

report! = order_not_pending

- OrderNotPendingError

$\Xi$ State

$i d$ ? : OrderId

report! : Report

orderStatus $(i d ?) \neq$ pending

report! = order_not_pending

scenario NotEnoughStockError

$i d ?=2_{a}, 9_{b}<77_{c}$

\begin{tabular}{|l|l|l|l|}
\hline stock & orders & orderStatus & freeIds \\
\hline$\{$ nut $\mapsto 5$, & $\{1 \mapsto\{$ nut $\mapsto 2$, bolt $\mapsto 2\}$, & $\{1 \mapsto$ invoiced,, & $\{3,4\}$ \\
bolt $\left._{d} \mapsto 9_{b}\right\}$ & $2_{a} \mapsto\left\{\right.$ bolt $\left.\left._{d} \mapsto 77_{c}\right\}\right\}$ & $2 \mapsto$ pending $\}$ & \\
\hline
\end{tabular}

report! = not_enough_stock

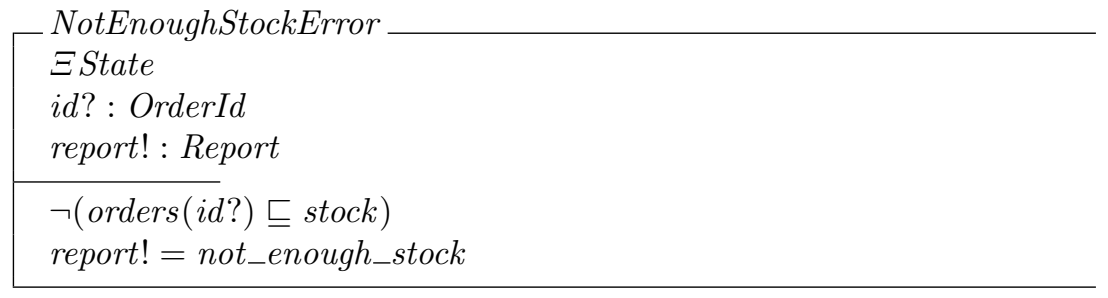


We define InvoiceOrderOp to deal with all situations. When multiple errors happen at the same time, the definition is unspecific about which error report to return. We will discuss nondeterminism in subsection 7.4.

$$
\begin{array}{r}
\text { InvoiceOrderOp }==\begin{array}{l}
\text { InvoiceOrder } \vee \text { IdNotFoundError } \vee \\
\text { OrderNotPendingError } \vee \text { NotEnoughStockError }
\end{array}
\end{array}
$$

\section{Cancel Order}

A pending order may be cancelled. Its order id is returned to the pool of free id's for future use.

scenario CancelOrder

$i d ?=2_{a}$

\begin{tabular}{|l|l|l|l|}
\hline stock & orders & orderStatus & freeIds \\
\hline$\{$ nut $\mapsto 5$, & $\{1 \mapsto\{$ nut $\mapsto 2$, bolt $\mapsto 2\}$, & $\{1 \mapsto$ invoiced, & $\{3,4\}$ \\
bolt $\mapsto 6\}$ & $2_{a} \mapsto\{$ bolt $\left.\mapsto 3\}\right\}$ & $2_{a} \mapsto$ pending \\
$b$ & \\
\hline$\ldots$ & $\{1 \mapsto\{$ nut $\mapsto 2$, bolt $\mapsto 2\}\}$ & $\{1 \mapsto$ invoiced $\}$ & $\left\{2_{a}, 3,4\right\}$ \\
\hline
\end{tabular}

report $!=O K$

The domain restriction symbol $\triangleleft$ is used to remove maplets for order id $2_{a}$ from orders and orderStatus.

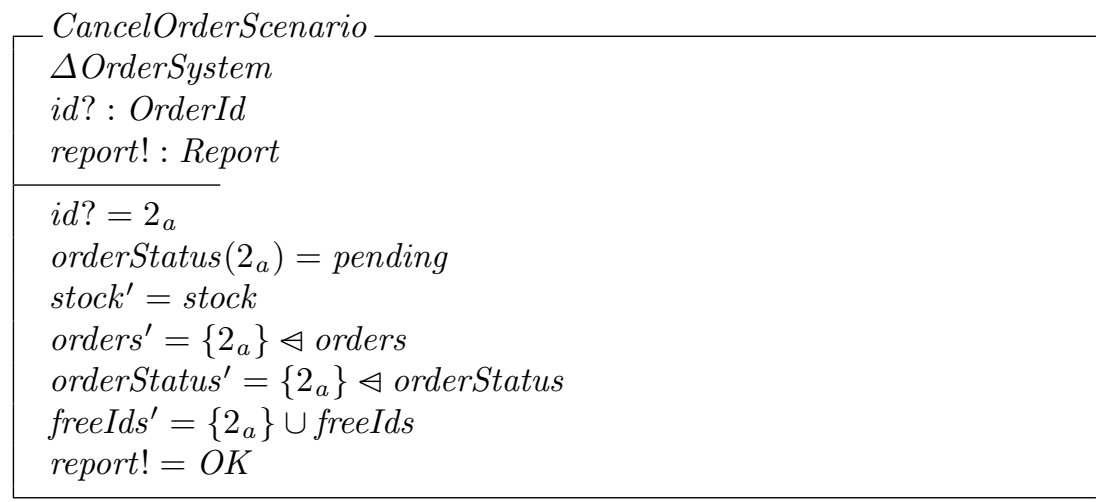

We generalise the scenario schema by replacing $2_{a}$ with $i d$ ?. After simplification, we have schema CancelOrder. 


\begin{tabular}{|c|}
\hline $\begin{array}{l}\Delta \text { OrderSystem } \\
i d ?: \text { OrderId } \\
\text { report! : Report }\end{array}$ \\
\hline $\begin{array}{l}\text { orderStatus }(\text { id } ?)=\text { pending } \\
\text { stock }{ }^{\prime}=\text { stock } \\
\text { orders }{ }^{\prime}=\{\text { id } ?\} \text { orders } \\
\text { orderStatus } s^{\prime}=\{\text { id? }\} \triangleleft \text { orderStatus } \\
\text { freeIds }{ }^{\prime}=\{i d ?\} \cup \text { freeIds } \\
\text { report } !=O K\end{array}$ \\
\hline
\end{tabular}

It is an error trying to cancel an order that does not exist or have already been invoiced. We can reuse error detecting schemas to handle all situations.

$$
\begin{gathered}
\text { CancelOrderOp }==\text { CancelOrder } \vee \text { IdNotFoundError } \vee \\
\text { OrderNotPendingError }
\end{gathered}
$$

\section{Enter Stock}

Entering stock is the task of replenishing depleted stock. By assuming that there is always sufficient storage space, we don't worry about detecting an error for this task. The postconditions concerning the updated stock quantities are expressed with the bag addition symbol $\uplus$.

scenario EnterStock

newStock $?=\left\{\right.$ nut $_{a} \mapsto 80_{b}$, bolt $\left._{c} \mapsto 70_{d}\right\}$

\begin{tabular}{|l|l|l|l|}
\hline stock & orders & orderStatus & freeIds \\
\hline$\left\{\right.$ nut $_{a} \mapsto 5_{e}$, & $\{1 \mapsto\{$ nut $\mapsto 2$, bolt $\mapsto 2\}$, & $\{1 \mapsto$ invoiced, & $\{3,4\}$ \\
bolt $\left._{c} \mapsto 9_{f}\right\}$ & $2 \mapsto\{$ bolt $\mapsto 3\}\}$ & $2 \mapsto$ pending $\}$ & \\
\hline$\left\{\right.$ nut $t_{a} \mapsto 85_{g}$, & $\ldots$ & $\ldots$ & $\cdots$ \\
bolt $\left._{c} \mapsto 79_{h}\right\}$ & & & \\
\hline
\end{tabular}

report $!=O K, 85_{g}=5_{e}+80_{b}, 79_{h}=9_{f}+70_{d}$

\begin{tabular}{|l} 
EnterStockScenario \\
$\Delta$ OrderSystem \\
newStock? : Order \\
report $!$ Report \\
\hline newStock $?=\left\{\right.$ nut $_{a} \mapsto 80_{b}$, bolt $\left._{c} \mapsto 70_{d}\right\}$ \\
stock $^{\prime}=$ stock $_{\text {nut }} \mapsto 80_{b}$, bolt $\left._{c} \mapsto 70_{d}\right\}$ \\
orders $^{\prime}=$ orders \\
orderStatus $^{\prime}=$ orderStatus \\
freeIds \\
report $!=$ OKeeIds
\end{tabular}




\begin{tabular}{|l} 
EnterStock \\
$\Delta$ OrderSystem \\
newStock? : Order \\
report $:$ Report \\
\hline stock $^{\prime}=$ stock $\uplus$ newStock $?$ \\
orders $^{\prime}=$ orders \\
orderStatus $^{\prime}=$ orderStatus \\
freeIds $=$ freeIds \\
report $!=$ OK
\end{tabular}

\section{Analysis}

\subsection{Validation}

We can apply the values of input parameters and pre-states in a scenario to its operation schema. If the post-state and output parameters obtained are the same as in the original scenario, we know that the operation schema works correctly for the scenario.

Another type of validation we can perform is to apply new input parameters and pre-state values to an operation schema. This exercise in essence creates new scenarios. If the customer is satisfied with the newly created post-states and output parameters, we gain confidence that our $\mathrm{Z}$ schemas meet the user requirements. For instance, we can validate the InvoiceOrder schema with the following input parameter and pre-state different from our scenarios.

$$
\begin{aligned}
& i d ?=3 \\
& \text { stock }=\{\text { nut } \mapsto 5, \text { bolt } \mapsto 9\} \\
& \text { orders }=\{1 \mapsto\{\text { nut } \mapsto 2\} \text {, } \\
& 2 \mapsto\{\text { nut } \mapsto 4, \text { bolt } \mapsto 3\}, \\
& 3 \mapsto\{\text { nut } \mapsto 5, \text { bolt } \mapsto 6\}\} \\
& \text { orderStatus }=\{1 \mapsto \text { invoiced }, 2 \mapsto \text { invoiced }, 3 \mapsto \text { pending }\} \\
& \text { freeIds }=\{4\}
\end{aligned}
$$

The first two predicates in the InvoiceOrder schema specify the preconditions of the schema. They both evaluate to true. The next four predicates specify the resulting values of the four tables. The last predicate specifies the output parameter. To save space, we only show the evaluation of two predicates below.

$$
\begin{aligned}
& \operatorname{orders}(i d ?) \sqsubseteq \text { stock } \\
& \text { orders }(3) \sqsubseteq\{\text { nut } \mapsto 5, \text { bolt } \mapsto 9\} \\
& \{\text { nut } \mapsto 5, \text { bolt } \mapsto 6\} \sqsubseteq\{\text { nut } \mapsto 5, \text { bolt } \mapsto 9\} \\
& \text { true } \\
& \text { stock }^{\prime}=\text { stock } \mapsto \text { orders }(\text { id } ?) \\
& \text { stock }^{\prime}=\{\text { nut } \mapsto 5, \text { bolt } \mapsto 9\} \uplus\{\text { nut } \mapsto 5, \text { bolt } \mapsto 6\} \\
& \text { stock }^{\prime}=\{\text { bolt } \mapsto 3\}
\end{aligned}
$$


After evaluating the schema predicates, we have the following new scenario. Though without subscripts, pre- and post-conditions, there is enough information for the customer to decide that the new scenario matches the expectation.

scenario InvoiceOrderNew
$i d ?=3$
\begin{tabular}{|l|l|r|l|}
\hline stock & orders & orderStatus & ids \\
\hline$\{$ nut $\mapsto 5$, & $\{1 \mapsto\{$ nut $\mapsto 2\}$, & $\{1 \mapsto$ invoiced, & $\{4\}$ \\
bolt $\mapsto 9\}$ & $2 \mapsto\{$ nut $\mapsto 4$, bolt $\mapsto 3\}$ & $2 \mapsto$ invoiced, & \\
& $3 \mapsto\{$ bolt $\mapsto 5$, bolt $\mapsto 6\}\}$ & $3 \mapsto$ pending $\}$ & \\
\hline$\{$ bolt $\mapsto 3\}$ & $\ldots$ & $\{1 \mapsto$ invoiced, & $\ldots$ \\
& & $2 \mapsto$ invoiced, & \\
& & $3 \mapsto$ invoiced $\}$ & \\
\hline
\end{tabular}

report $!=O K$

\subsection{Underspecification}

Underspecification happens when a required condition is missing. Suppose we had omitted the following precondition of checking for sufficient stock in schema InvoiceOrder.

$$
\operatorname{orders}(i d ?) \sqsubseteq \text { stock }
$$

If we validate the schema with excessive ordered quantities, the operation will still succeed. The customer can easily spot that the new scenario should have caused an error.

\subsection{Overspecification}

Overspecification happens when unnecessary conditions are included in a scenario. Recall scenario NewOrder. The quantities of the nuts and bolts in the input parameter were both 4 by coincidence. In our original scenario, they have different subscripts $b$ and $d$ to indicate that they need not be the same. Suppose we had made a mistake by using the same subscript $b$ on both occurrences of 4 . We would have a slightly different scenario.

scenario OverSpecifiedNewOrder

order $?=\left\{\right.$ nut $_{a} \mapsto 4_{b}$, bolt $\left._{c} \mapsto 4_{b}\right\}$

\begin{tabular}{|c|c|c|c|}
\hline stock & orders & orderStatus & freeIds \\
\hline $\begin{aligned}\{\text { nut } & \mapsto 5 \\
\text { bolt } & \mapsto 6\}\end{aligned}$ & $\begin{aligned}\{1 & \mapsto\{\text { nut } \mapsto 2, \text { bolt } \mapsto 2\}, \\
2 & \mapsto\{\text { bolt } \mapsto 3\}\}\end{aligned}$ & $\left\{\begin{array}{r}\{1 \mapsto \text { invoiced } \\
2 \mapsto \text { invoiced }\}\end{array}\right.$ & $\left\{3_{e}, 4\right\}$ \\
\hline . & $\begin{aligned}\{1 & \mapsto\{\text { nut } \mapsto 2, \text { bolt } \mapsto 2\}, \\
2 & \mapsto\{\text { bolt } \mapsto 3\}, \\
3_{e} & \left.\mapsto\left\{\text { nut }_{a} \mapsto 4_{b}, \text { bolt }_{c} \mapsto 4_{b}\right\}\right\}\end{aligned}$ & $\begin{array}{l}\{1 \mapsto \text { invoiced } \\
2 \mapsto \text { invoiced } \\
\left.3_{e} \mapsto \text { pending }\right\}\end{array}$ & $4\}$ \\
\hline
\end{tabular}

$i d !=3_{e}$, report $!=O K$ 
The equality implied by the identical subscripts gives rise to the first predicate in the following schema. When we validate the schema with order? $=\{n u t \mapsto$ 7 , bolt $\mapsto 9\}$, the new predicate would evaluate to false. Overspecification is caught when the operation fails on legitimate input and pre-state.

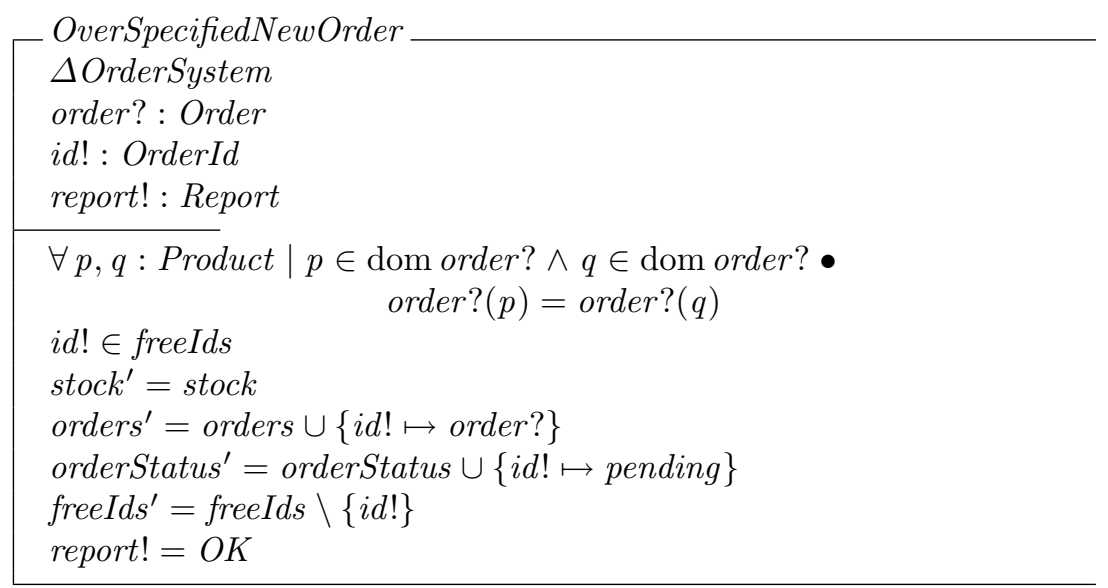

\subsection{Nondeterminism}

Recall that we have defined InvoiceOrderOp to catch errors.

$$
\begin{array}{r}
\text { InvoiceOrderOp }==\text { InvoiceOrder } \vee \text { IdNotFoundError } \vee \\
\text { OrderNotPendingError } \vee \text { NotEnoughStockError }
\end{array}
$$

What if the preconditions of OrderNotPendingError and NotEnoughStockError are true at the same time? Which error report are we going to get? In this nondeterministic definition of InvoiceOrderOp, we could get either one. If it is necessary to distinguish the two errors, we can strengthen their preconditions so that their conjunction will always be false. This is the general strategy we could use to eliminate nondeterminism.

\subsection{Tool Support}

The success of a methodology relies heavily on the availability of good tools. There are many Z tools around, for example, CADiZ, CZT(Community Z Tools), Z/EVES, and ZETA. They can be used to check for type conisistency and prove theorems about a specification. Some allow you to validate operation schemas against scenarios as we just did [11]. But to use precise scenarios in a practical setting, we need native support to keep track of subscripts, validate scenarios against schemas and maintain traceability of schemas to their sourcing scenarios. 


\section{Conclusion}

The $\mathrm{Z}$ specification we derived in this paper is almost identical to the one found in [10]. One notable difference is that our specification catches more errors. It is premature to conclude how the use of precise scenarios would shape a $\mathrm{Z}$ specification. However we do not seem to have lost any capability to create a generic $\mathrm{Z}$ specification.

What evidence do we have in support of our claim that precise scenarios bridge the gap between customers and formal specifications? Our precise scenario descriptions use simple $\mathrm{Z}$ concepts, namely input, output, maplet, set enumeration, pre-state and post-state. On the other hand, schemas use additional Z concepts like set membership, set union, set difference, sub-bag, bag difference, override, domain restriction, and bag addition. Due to the smaller number of symbols used, the customers would have an easier time learning to read precise scenarios. Our experience on other problems taken from the areas of numerical computation, sorting and telephone system has been similar in that precise scenarios are representable concisely with significantly less number of concepts than schemas. Biologists and educationists also suggest that understanding begins with concrete examples [12]. We expect the majority of customers to prefer dealing with concrete examples rather than abstract descriptions.

The use of precise scenarios for the development and validation of schemas relates to a software testing technique called equivalence partitioning [13]. The technique ensures that a scenario is selected from every class of similar situations. For example, the operation InvoiceOrder can be partitioned into at least two classes of situations, one for ordered quantities being a sub-bag of stock quantities and one for otherwise. If necessary, we can divide them into more partitions. For example, we can add a class of situations that the ordered quantities are identical to the stock qunatities. Why is testing necessary in the application of formal methods? Refinement can produce an implementation guaranteed to be correct according to a formal specification. Therefore the research community has long held the attitude that formal methods reduce or eliminate the need of testing. But how do we know the formal specification is complete in the first place? Even for toy problems, it is hard to be certain that a formal specification is complete. Though the use of precise scenarios cannot guarantee completeness, the improved customer involvement can only help.

Formal methods have been focusing on the initial creation of an abstract specification and its gradual refinement to concrete implementation. Our proposal challenges the validity of this conventional wisdom. We suggest an approach to

precede the current formal approaches by concrete examples used as a basis for the abstract specification. The precise scenarios has a dual purpose; serving as a solid foundation for a formal specification and a bridge between the customers to the formal specification.

The current paper shows only scenarios with pre- and post-states. Work in progress includes longer scenarios with intermediate states. We have started working on alternative representations for the precise scenarios to facilitate the 
derivation of specifications in languages other than Z. Eventually, we would like to field-test the approach when suitable tool support becomes available.

\section{References}

1. The Standish Group: The CHAOS Report (1994) 5

2. Zimmerman, M.K., Lundqvist, K., Leveson, N.: Investigating the Readability of State-Based Formal Requirements Specification Languages. ICSE'02: 24th International Conference on Software Engineering, May (2002) 33-43

3. Glass, R.L.: The Mystery of Formal Methods Disuse. Communications of the ACM, Vol. 47, No. 8 (2004) 15-17

4. Amyot, D., Logrippo, L., Buhr, R.J.A., Gray, T.: Use Case Maps for the Capture and Validation of Distributed Systems Requirements. RE'99: 4th IEEE International Symposium on Requirements Engineering, June (1999) 44-54

5. Whittle, J., Schumann, J.: Generating Statechart Designs from Scenarios. ICSE'00: 22nd International Conference on Software Engineering, June (2000) 314-323

6. Uchitel, S., Kramer, J., Magee, J.: Synthesis of Behavioral Models from Scenarios. IEEE Transactions on Software Engineering, vol. 29, no. 2 (2003) 99-115

7. Damas, C., Lambeau, B., Dupont, P., van Lamsweerde, A.: Generating Annotated Behaviour Models from End-User Scenarios. IEEE Transactions on Software Engineering, vol. 31, no. 12 (2005) 1056-1073

8. Grieskamp, W., Lepper, M.: Using Use Cases in Executable Z. Liu, S., McDermid, J.A., Hinchey, M.G. (ed.) ICFEM 2000: 3rd IEEE International Conference on Formal Engineering Methods, September (2000) 111-119

9. Büssow, R., Grieskamp, W.: A Modular Framework for the Integration of Heterogenous Notations and Tools. Araki, K., Galloway, A., Taguchi, K. (ed.) IFM99: 1st International Conference on Integrated Formal Methods, June (1999) 211-230

10. Bowen, J.P.: Chapter 1 - Z. Habrias, H., Frappier M. (ed.): Software Specification Methods, ISTE (2006) 3-20

11. Saaltink, M.: The Z/EVES 2.0 User's Guide. TR-99-5493-06a, ORA Canada (1999) 31-32

12. Zull, J.E.: The Art of Changing the Brain. Stylus Publishing (2002) 102-103

13. Sommerville, I.: Software Engineering, 6th Edition. Addison Wesley (2001) 444-447 